\title{
Ocorrência de distúrbios na coluna e fatores associados em idosos: estudo populacional em município do extremo Sul do Brasil
}

\author{
Occurrence of spinal disorders and associated factors \\ among the elderly: a population study in a municipality \\ in the deep south of Brazil
}

Mirelle de Oliveira Saes (https://orcid.org/0000-0001-7225-1552) ${ }^{1}$

Juliana Damasceno Nunes Lopes (https://orcid.org/0000-0002-3721-1042) ${ }^{2}$

Bruno Pereira Nunes (http://orcid.org/0000-0002-4496-4122) ${ }^{2}$

Suele Manjourany Silva Duro (https://orcid.org/0000-0001-5730-0811) ${ }^{2}$

Luiz Augusto Facchini (https://orcid.org/0000-0002-5746-5170) ${ }^{3}$

Elaine Thumé (https://orcid.org/0000-0002-1169-8884) ${ }^{2}$
${ }^{1}$ Faculdade de Medicina, Universidade Federal do Rio Grande. R. Visconde de Paranaguá 102, Campus da Saúde. 96203-900 Rio Grande RS Brasil. mirelleosaes@gmail.com

${ }^{2}$ Faculdade de Enfermagem, Universidade Federal de Pelotas (UFPel). Pelotas RS Brasil.

${ }^{3}$ Programa de PósGraduação em Epidemiologia, UFPel. Pelotas RS Brasil.

\begin{abstract}
The scope of this study was to estimate the prevalence of spinal disorders among the elder$l y$ and analyze potential associated factors. It involved a cross-sectional study of a sample of 1,593 elderly individuals aged 60 years or more residing in the urban area of Bagé, State of Rio Grande do Sul. In addition to the "spinal disorders" outcome, demographic, socioeconomic and behavioral variables, health perception, functional activities and the use of health services were investigated. Data collection was conducted by means of face-to-face interviews. Poisson regression analysis with robust variance was used to verify the factors associated with the outcome analyzed. Of the 1,593 participants, $37.4 \%$ reported spinal disorders. The factors related to the outcome were: low level of schooling, poor self-assessment of health, consultation in the last three months and the presence of hypertension, rheumatism and fractures. Interventions for musculoskeletal health can contribute to reduce the damages caused by spinal disorders among the elderly, such as loss of functional capacity, increased demands for health care and reduced quality of life.
\end{abstract}

Key words Elderly, Spine, Back pain
Resumo Estimar a prevalência de distúrbios na coluna em idosos e analisar potenciais fatores associados. Estudo de delineamento transversal em uma amostra de 1.593 idosos com 60 anos ou mais residentes na zona urbana do município de Bagé-RS. Além do desfecho "distúrbios na coluna vertebral", foram investigadas variáveis demográficas, socioeconômicas, comportamentais, percepção de saúde, atividades funcionais e utilização de serviços de saúde. A coleta de dados foi realizada por meio de entrevista face a face. Análise de regressão de Poisson com variância robusta foi utilizada para verificar os fatores associados ao desfecho analisado. Dos 1.593 participantes, 37,4\% referiram distúrbios na coluna vertebral. Os fatores relacionados ao desfecho foram; pertencer ao sexo feminino, baixa escolaridade, autoavaliação ruim de saúde, consulta nos últimos três meses e presença de hipertensão, reumatismo e fraturas. Ações de intervenção para saúde musculoesquelética podem contribuir para redução dos danos decorrentes distúrbios na coluna em idosos, como perda da capacidade funcional, aumento das demandas de cuidados em saúde e redução da qualidade de vida.

Palavras-chave Idoso, Coluna, Dor nas costas 


\section{Introdução}

Os distúrbios da coluna vertebral (DCV) caracterizam-se como uma ampla e heterogênea variedade de doenças que afetam as vértebras, discos vertebrais, articulações facetárias, tendões, ligamentos, músculos, medula espinhal e raízes nervosas, cuja maioria se manifesta como dor ${ }^{1}$. Alterações degenerativas na coluna vertebral levam a alterações bioquímicas, estruturais e biomecânicas que resultam em dor e incapacidade ${ }^{2}$.

A dor nas costas (DC) ocasionada por DCV é considerada a segunda doença crônico-degenerativa mais prevalente entre a população idosa e contribui para o declínio na capacidade funcional, o aumento na demanda aos serviços de saúde e despesas com saúde com consequências na redução da qualidade de vida ${ }^{3,4}$. A prevalência internacional de DC varia entre 12 a $33 \% \%^{4-6}$, enquanto no Brasil cerca de $30 \%$ dos idosos apresentam dor nas costas autorreferida ${ }^{7}$.

Diferentes fatores podem estar associados a DCV, entre eles os biológicos e socioeconômicos como: sexo feminino, comorbidades, incapacidade funcional, baixos níveis educacional e de ren$\mathrm{da}$, e os comportamentais: tabagismo, obesidade, alcoolismo e comportamento sedentário ${ }^{6-10}$.

Contudo, há escassez de estudos sobre o desfecho DCV, particularmente no Brasil. Geralmente, os estudos optam por investigar a presença do problema nas regiões da coluna, como, por exemplo, dor lombar ou dor cervical. Portanto, as lacunas na literatura se referem a avaliação de DCV, abordando uma única estrutura musculoesquelética que pode apresentar mais de uma das suas regiões comprometidas concomitantemente, podendo diferir quanto aos fatores associados e necessidade de intervenção.

Neste contexto, este artigo tem como objetivo estimar a prevalência de DCV e analisar os potenciais fatores associados na população idosa de Bagé, Rio Grande do Sul (RS), Brasil.

\section{Metodologia}

Trata-se de um estudo de delineamento transversal de base populacional, com dados coletados de julho a novembro de 2008, em uma amostra de idosos com 60 anos ou mais, residentes na zona urbana e localizados a partir da área de abrangência dos serviços de atenção básica à saúde do município de Bagé-RS.

A coleta de dados foi realizada respeitando as 20 Unidades Básicas de Saúde (UBS) que o município dispunha, sendo 15 da Estratégia de Saúde da Família (ESF) e cinco Unidades Básicas Tradicionais. A ESF apresentava uma cobertura de $51 \%$ da população urbana. Para localização da amostra, a área de abrangência das UBS foi dividida em microáreas, com identificação numérica de cada quadra, sorteando aleatoriamente o ponto inicial de coleta de dados. Com o intuito de garantir que todos os domicílios tivessem a mesma probabilidade de compor a amostra, foi empregado o pulo sistemático de uma a cada cinco residências. Todos os moradores com 60 anos ou mais de idade residentes nos domicílios selecionados foram convidados a participar do estudo. As entrevistas não realizadas após três tentativas em dias e horários diferentes foram consideradas perdas e/ou recusas, não havendo substituição. Indivíduos que, no momento da entrevista, encontravam-se em viagem, privados de liberdade por decisão judicial ou residindo em instituições de longa permanência não atenderam os critérios de inclusão. No caso de pessoas sem condições físicas ou mentais para responder o questionário, este foi aplicado ao cuidador principal.

Os dados foram coletados por entrevistadores previamente treinados através da aplicação de questionário estruturado com questões précodificadas. O tamanho de amostra foi calculado considerando $10 \%$ de perdas e recusas, e um efeito de delineamento de $1.3,80 \%$ de poder para detectar riscos relativos de 1.5 e exposições que afetavam, no mínimo, $4 \%$ da população ${ }^{11}$.

O desfecho "distúrbios na coluna vertebral" foi investigado através da variável "Algum médico disse que o(a) Sr.(a) tem doença na coluna?". As variáveis independentes incluídas foram: sexo (feminino e masculino), idade em anos completos (60 a $64 ; 65$ a $69 ; 70$ a $74 ; 75$ ou mais), residir sozinho (sim/não), cor da pele auto-referida (branca; preta; parda/indígena/amarela), situação conjugal (casado ou com companheiro; viúvo; solteiro ou separado), escolaridade em anos completos (nenhum, 1 a 7, 8 ou mais), classificação econômica segundo Associação Brasileira de Empresas de Pesquisa - ABEP (A/B, C, D/E), uso de tabaco (ex-fumante, fumante, nunca fumou), uso de álcool nos últimos 30 dias ( $\mathrm{sim} /$ não), comportamento sedentário: assistir TV $>3$ horas por dia (sim/não), autoavaliação de saúde (ótimo/bom, regular e ruim/péssimo), sentimento com relação à vida (satisfeito/insatisfeito), comparado com outras pessoas de sua idade, sua saúde está (melhor, igual, pior), história de queda no último ano (sim/não), história de fraturas no último ano (sim/não), diagnóstico médico de 
hipertensão arterial sistêmica (HAS) (sim/não), diabetes mellitus (DM) (sim/não), diagnóstico médico de reumatismo, artrite ou artrose ( $\mathrm{sim} /$ não), déficit cognitivo (mini-exame mental: até $22 / 23$ pontos; sim/não), incapacidade funcional (necessidade de ajuda parcial ou total para pelo menos uma das atividades investigadas; atividades básicas - Índice de Katz e instrumentais da vida diária - Escala de Lawton e Brody, ambos validados no Brasil), utilização de serviço de urgência e/ou domiciliar e/ou consulta médica nos últimos 03 meses (sim/não) e utilização de serviço hospitalar nos últimos 12 meses ( $\operatorname{sim} /$ não).

As análises foram conduzidas no programa Stata versão 12.1. Utilizou-se a estatística descritiva com o cálculo das prevalências e respectivos intervalos de confiança (IC95\%). Na análise bruta foram empregados testes de qui-quadrado para heterogeneidade ou tendência linear. A análise ajustada foi realizada com o objetivo de avaliar a associação do desfecho com as variáveis independentes, controlando os possíveis fatores de confusão e as variáveis de exposição foram organizadas de acordo com o seguinte modelo hierárquico: $1^{\circ}$ nível - Características demográficas e socioeconômicas; $2^{\circ}$ nível - Características comportamentais; $3^{\circ}$ nível - Percepção de saúde e situação de saúde, $4^{\circ}$ nível - Atividades básicas (ABVD) e instrumentais (AIVD) da vida diária; e $5^{\circ}$ nível - Utilização de serviços de saúde. Utilizou-se ajuste backward por níveis hierárquicos, através da regressão de Poisson com ajuste robusto da variância. Foram calculadas as razões de prevalência e respectivos intervalos de confiança (IC95\%) e mensurados os valores p do teste de Wald de heterogeneidade e tendência linear. Associações com valor- $\mathrm{p}<0,05$ foram consideradas estatisticamente significativas.

A pesquisa foi aprovada pelo Comitê de Ética em Pesquisa da Faculdade de Medicina da Universidade Federal de Pelotas. Os princípios éticos foram resguardados a partir da assinatura dos participantes no Termo de Consentimento Livre e Esclarecido e da garantia do anonimato absoluto.

\section{Resultados}

Foram investigados 1.593 idosos, perdas representaram $4,0 \%$ e recusas $3,0 \%$. Com relação às características da amostra, observou-se que a maioria era do sexo feminino $(63,0 \%)$, com idade de 75 anos ou mais $(31,2 \%)$, de cor da pele branca $(78,6 \%)$ e referiam ter de 1 a 7 anos de estudo (54,6\%). Além disso, 51,2\% da amostra era casada ou vivia com companheiro, $17,5 \%$ dos idosos residiam sozinhos, $38,4 \%$ pertenciam à classificação econômica C e 34,0\% à D/E.

Quanto às variáveis comportamentais, 39,6\% relataram ter parado de fumar, $16,0 \%$ consumiram álcool nos últimos 30 dias e mais da metade $(59,3 \%)$ admitiram assistir 3 ou mais horas diárias de televisão. No que se refere à auto-avaliação de saúde, 34,1\% a consideravam regular, $94,3 \%$ diziam estar satisfeitos com a vida e 44,6\% dos entrevistados consideravam sua saúde igual a das outras pessoas.

Ao investigar as doenças, verificou-se que $4,0 \%$ dos idosos relataram ter tido algum tipo de fratura e cerca de 30,0\% referiram quedas no último ano. $\mathrm{O}$ diagnóstico médico de hipertensão arterial sistêmica e de diabetes mellitus foram referidos por 55,3\% e 15,1\% dos idosos entrevistados, respectivamente. Quase um terço dos idosos $(27,2 \%)$ possuía diagnóstico médico de reumatismo/artrite/artrose, enquanto $9,8 \%$ referiram ter tido acidente vascular encefálico e $34,1 \%$ possuíam déficit cognitivo. A prevalência de incapacidade para atividades básicas da vida diária foi de $10,6 \%$ e para atividades instrumentais de 34,2\%. Com relação à utilização dos serviços de saúde, $17,7 \%$ dos entrevistados referiram ter se hospitalizado nos últimos 12 meses, $12,8 \%$ relataram ter ido à consulta de emergência, 54,5\% a consulta médica e $6,8 \%$ dos idosos referiram ter recebido algum tipo de atendimento domiciliar nos últimos três meses, anteriores à entrevista (Tabela 1).

A prevalência de idosos com distúrbios na coluna vertebral foi de $37,4 \%$, destes $91,5 \%$ referiu ter o problema há mais de 12 meses. $\mathrm{Na}$ análise bruta observou-se que as variáveis independentes sexo, idade, estado civil, comportamento sedentário, autoavaliação de saúde, sentimento com relação a vida, fraturas, queda no último ano, HAS, DM, reumatismo, déficit cognitivo, ABVD e consulta nos últimos três meses estiveram associadas com o desfecho pesquisado (Tabela 2).

$\mathrm{Na}$ análise ajustada os resultados revelaram uma probabilidade de distúrbios na coluna $58 \%$ (RP: 1,58; IC95\% 1,36-1,83) maior no sexo feminino, em comparação a homens e 30\% (RP: 1,31; IC95\% 1,10-1,56) maior em indivíduos com 1 a 7 anos ou nenhuma escolaridade, comparados aos idosos com 8 anos ou mais de estudo. O estado civil mostrou ser um fator de proteção para distúrbios na coluna entre os idosos solteiros/ separados (RP: 0,77; IC95\% 0,64-0,94) e viúvos (RP: 0,75; IC95\% 0,65-0,88). O comportamento sedentário, caracterizado por assistir televisão 
Tabela 1. Análise descritiva das variáveis independentes dos idosos de Bagé, RS, 2008 ( N = 1.593).

\begin{tabular}{|c|c|c|}
\hline Variáveis & $\mathbf{N}$ & $\%$ \\
\hline \multicolumn{3}{|l|}{ Sociodemográficas } \\
\hline \multicolumn{3}{|l|}{ Sexo } \\
\hline Masculino & 593 & 37,2 \\
\hline Feminino & 1.000 & 63,0 \\
\hline \multicolumn{3}{|l|}{ Idade } \\
\hline 60 a 64 anos & 400 & 25,1 \\
\hline 65 a 69 anos & 374 & 23,5 \\
\hline 70 a 74 anos & 322 & 20,2 \\
\hline 75 ou mais & 497 & 31,2 \\
\hline \multicolumn{3}{|l|}{ Cor da pele } \\
\hline Branca & 1.252 & 78,6 \\
\hline Preta & 139 & 8,7 \\
\hline Parda/indígena/amarela & 202 & 12,7 \\
\hline \multicolumn{3}{|l|}{ Situação Conjugal } \\
\hline Casado ou com companheiro & 816 & 51,2 \\
\hline Solteiro/separado & 238 & 14,9 \\
\hline Viúvo & 538 & 33,8 \\
\hline \multicolumn{3}{|l|}{ Reside sozinho } \\
\hline Não & 1.313 & 82,4 \\
\hline $\operatorname{Sim}$ & 280 & 17,6 \\
\hline \multicolumn{3}{|l|}{ Escolaridade } \\
\hline Nenhum & 372 & 23,6 \\
\hline 1 a 7 anos & 858 & 54,6 \\
\hline 8 ou mais anos & 342 & 21,7 \\
\hline \multicolumn{3}{|l|}{ Classe econômica } \\
\hline $\mathrm{A} / \mathrm{B}$ & 429 & 27,1 \\
\hline $\mathrm{C}$ & 615 & 38,9 \\
\hline $\mathrm{D} / \mathrm{E}$ & 537 & 34,0 \\
\hline \multicolumn{3}{|l|}{ Comportamentais } \\
\hline \multicolumn{3}{|l|}{ Fumante } \\
\hline Não, nunca fumou & 717 & 45,0 \\
\hline Sim, fuma & 244 & 15,3 \\
\hline Já fumou, mas parou & 631 & 39,6 \\
\hline \multicolumn{3}{|l|}{ Bebida } \\
\hline Sim & 254 & 16,0 \\
\hline Não & 1.330 & 84,0 \\
\hline \multicolumn{3}{|l|}{ Comportamento sedentário } \\
\hline$<3$ horas & 595 & 40,7 \\
\hline 3 horas ou mais & 882 & 59,3 \\
\hline \multicolumn{3}{|l|}{ Percepção de saúde } \\
\hline \multicolumn{3}{|l|}{ Auto avaliação } \\
\hline Péssimo & 49 & 3,2 \\
\hline Ruim & 60 & 3,9 \\
\hline Regular & 525 & 34,1 \\
\hline Bom & 692 & 44,9 \\
\hline Ótimo & 214 & 13,9 \\
\hline
\end{tabular}

Tabela 1. Análise descritiva das variáveis independentes dos idosos de Bagé, RS, 2008 ( N = 1.593).

\begin{tabular}{lrr}
\hline \multicolumn{1}{c}{ Variáveis } & N & \multicolumn{1}{c}{$\%$} \\
\hline Sentimento com relação a vida & & \\
$\quad$ Insatisfeito & 87 & 5,6 \\
Satisfeito & 1.449 & 94,3 \\
Comparado a outra pessoa & & \\
$\quad$ Melhor & 684 & 44,6 \\
Igual & 684 & 44,6 \\
Pior & 164 & 10,7 \\
Doenças & & \\
Fratura & & \\
Não & 1.528 & 95,6 \\
Sim & 64 & 4,0 \\
Quedas no último ano & & \\
Não & 1.145 & 72,0 \\
Sim & 446 & 28,0 \\
Hipertensão & & \\
Não & 712 & 44,7 \\
Sim & 881 & 55,3 \\
Diabetes & & \\
Não & 1.352 & 84,9 \\
Sim & 241 & 15,1 \\
Reumatismo/artrite/artros & &
\end{tabular}

Reumatismo/artrite/artrose

$\begin{array}{lrr}\text { Não } & 1.158 & 72,7 \\ \text { Sim } & 434 & 27,2 \\ \text { Acidente Vascular Encefálico } & & \\ \quad \text { Não } & 1.436 & 90,1 \\ \text { Sim } & 157 & 9,8 \\ \text { Déficit Cognitivo } & & \\ \quad \text { Sem déficit } & 998 & 65,9 \\ \text { Com déficit } & 516 & 34,1\end{array}$

\section{Capacidade funcional}

ABVD* $^{*}$

Sem dificuldade $\quad 1.424 \quad 89,4$

Com dificuldade $\quad 169 \quad 10,6$

AIVD\#

$\begin{array}{lll}\text { Sem dificuldade } & 1.048 & 65,8\end{array}$

Com dificuldade $\quad 545 \quad 34,2$

Utilização de serviços de saúde

Hospitalização 12 meses

$\begin{array}{lrr}\text { Não } & 1.310 & 82,3 \\ \text { Sim } & 282 & 17,7\end{array}$

Emergência 3 meses

$\begin{array}{lrr}\text { Não } & 1.387 & 87,2 \\ \text { Sim } & 204 & 12,8 \\ \text { Domicílio 3 meses } & & \\ \text { Não } & 1.482 & 93,1 \\ \text { Sim } & 109 & 6,8\end{array}$

Consulta 3 meses

$\begin{array}{lll}\text { Não } & 723 & 45,4\end{array}$

\begin{tabular}{l}
$\mathrm{Sim}$ \\
$868 \quad 54,5$ \\
\hline
\end{tabular}

^Atividades básicas de vida diárias; \#Atividades instrumentais de vida diária. 
Tabela 2. Prevalência de distúrbio na coluna vertebral, análise bruta e ajustada conforme as variáveis associadas em idosos de Bagé, RS, 2008 ( $\mathrm{N}=1.593)$.

\begin{tabular}{|c|c|c|c|c|c|c|c|}
\hline \multirow[t]{2}{*}{ Níveis } & \multirow[t]{2}{*}{ Variáveis } & \multicolumn{2}{|c|}{$\begin{array}{c}\text { Distúrbio } \\
\text { Coluna }\end{array}$} & \multicolumn{2}{|c|}{ Análise Bruta } & \multicolumn{2}{|c|}{ Análise Ajustada } \\
\hline & & Sim & $\%$ & RP (IC95\%) & $\mathbf{P}$ & RP (IC95\%) & $\mathbf{P}$ \\
\hline \multirow[t]{28}{*}{$1^{\circ}$ nível } & Sociodemográficas & & & & & & \\
\hline & Sexo & & & & 0,000 & & 0,000 \\
\hline & Masculino & 176 & 29,7 & 1 & & 1 & \\
\hline & Feminino & 419 & 42,0 & $1,41(1,22-1,63)$ & & $1,58(1,36-1,83)$ & \\
\hline & Idade & & & & 0,030 & & 0,150 \\
\hline & 60 a 64 anos & 154 & 38,5 & 1 & & 1 & \\
\hline & 65 a 69 anos & 159 & 42,6 & $1,10(0,93-1,31)$ & & $1,06(0,90-1,26)$ & \\
\hline & 70 a 74 anos & 119 & 36,9 & $0,96(0,79-1,16)$ & & $0,96(0,80-1,17)$ & \\
\hline & 75 ou mais & 163 & 32,9 & $0,85(0,71-1,02)$ & & $0,86(0,71-1,04)$ & \\
\hline & Cor da pele & & & & 0,372 & & 0,470 \\
\hline & Branca & 474 & 37,9 & 1 & & 1 & \\
\hline & Preta & 44 & 31,6 & $0,83(0,64-1,07)$ & & $0,85(0,66-1,09)$ & \\
\hline & Parda/indígena/amarela & 77 & 38,1 & $1,00(0,83-1,21)$ & & $0,98(0,81-1,18)$ & \\
\hline & Situação conjugal & & & & 0,045 & & 0,000 \\
\hline & $\begin{array}{l}\text { Casado ou com } \\
\text { companheiro }\end{array}$ & 329 & 40,4 & 1 & & 1 & \\
\hline & Solteiro/separado & 83 & 34,9 & $0,86(0,71-1,04)$ & & $0,77(0,64-0,94)$ & \\
\hline & Viúvo & 183 & 34,1 & $0,84(0,73-0,97)$ & & $0,75(0,65-0,88)$ & \\
\hline & Escolaridade & & & & $<0,021$ & & 0,000 \\
\hline & 8 ou mais anos & 106 & 31,0 & 1 & & 1 & \\
\hline & 1 a 7 anos & 342 & 39,9 & $1,28(1,07-1,53)$ & & $1,31(1,10-1,56)$ & \\
\hline & Nenhum & 140 & 37,7 & $1,21(0,99-1,49)$ & & $1,30(1,06-1,60)$ & \\
\hline & Classe econômica & & & & 0,582 & & 0,995 \\
\hline & $\mathrm{A} / \mathrm{B}$ & 156 & 36,4 & 1 & & 1 & \\
\hline & $\mathrm{C}$ & 235 & 38,2 & $1,05(0,89-121)$ & & $1,00(0,83-1,18)$ & \\
\hline & $\mathrm{D} / \mathrm{E}$ & 200 & 37,3 & $1,02(0,86-1,21)$ & & $0,99(0,81-1,20)$ & \\
\hline & Reside sozinho & & & & 1,121 & & 0,678 \\
\hline & Não & 502 & 38,3 & 1 & & 1 & \\
\hline & Sim & 93 & 33,2 & $0,86(0,72-1,03)$ & & $0,96(0,79-1,16)$ & \\
\hline \multirow[t]{11}{*}{$2^{\circ}$ nível } & Comportamentais & & & & & & \\
\hline & Fumante & & & & 0,483 & & 0,738 \\
\hline & Não, nunca fumou & 279 & 39,0 & 1 & & 1 & \\
\hline & Sim, fuma & 87 & 35,7 & $0,91(0,75-1,10)$ & & $1,04(0,85-1,27)$ & \\
\hline & Já fumou, mas parou & 229 & 36,3 & $0,93(0,81-1,06)$ & & $1,05(0,91-1,23)$ & \\
\hline & Bebida & & & & 0,103 & & 0,638 \\
\hline & Sim & 83 & 32,7 & 1 & & 1 & \\
\hline & Não & 508 & 38,3 & $0,85(0,70-1,03)$ & & $0,95(0,78-1,16)$ & \\
\hline & Comport. sedentário & & & & 0,013 & & 0,006 \\
\hline & $<3$ horas & 248 & 71,7 & 1 & & 1 & \\
\hline & 3 horas ou mais & 306 & 35,3 & $0,84(0,74-0,96)$ & & $0,83(0,73-0,94)$ & \\
\hline \multirow[t]{8}{*}{$3^{\circ}$ nível } & Percepção Saúde & & & & & & \\
\hline & Auto avaliação & & & & 0,000 & & 0,000 \\
\hline & Bom/Ótimo & 284 & 31,4 & 1 & & 1 & \\
\hline & Regular & 233 & 44,5 & $1,41(1,23-1,62)$ & & $1,29(1,12-1,49)$ & \\
\hline & Péssimo/ruim & 65 & 59,6 & $1,90(1,58-2,27)$ & & $1,95(1,60-2,38)$ & \\
\hline & Sentimento com rel. Vida & & & & 0,047 & & 0,923 \\
\hline & Insatisfeito & 41 & 47,1 & 1 & & 1 & \\
\hline & Satisfeito & 539 & 37,2 & $0,79(0,62-0,99)$ & & $0,98(0,77-1,25)$ & \\
\hline
\end{tabular}


Tabela 2. Prevalência de distúrbio na coluna vertebral, análise bruta e ajustada conforme as variáveis associadas em idosos de Bagé, RS, 2008 ( $\mathrm{N}=1.593)$.

\begin{tabular}{|c|c|c|c|c|c|c|c|}
\hline \multirow[t]{2}{*}{ Níveis } & \multirow[t]{2}{*}{ Variáveis } & \multicolumn{2}{|c|}{$\begin{array}{c}\text { Distúrbio } \\
\text { Coluna }\end{array}$} & \multicolumn{2}{|c|}{ Análise Bruta } & \multicolumn{2}{|c|}{ Análise Ajustada } \\
\hline & & Sim & $\%$ & RP (IC95\%) & $\mathbf{P}$ & RP (IC95\%) & $\mathbf{P}$ \\
\hline & Comparado. a outra pessoa & & & & 0,527 & & 0,156 \\
\hline & Melhor & 267 & 39,1 & 1 & & 1 & \\
\hline & Igual & 247 & 36,2 & $0,92(0,80-1,06)$ & & $0,93(0,81-1,06)$ & \\
\hline & Pior & 63 & 38,4 & $0,98(0,79-1,21)$ & & $0,80(0,64-1,01)$ & \\
\hline & \multicolumn{7}{|l|}{ Doenças } \\
\hline & Fraturas no último ano & & & & 0,003 & & 0,050 \\
\hline & Não & 561 & 36,8 & 1 & & 1 & \\
\hline & Sim & 34 & 53,1 & $1,44(1,13-1,83)$ & & $1,27(1,00-1,62)$ & \\
\hline & Queda no último ano & & & & 0,000 & & 0,125 \\
\hline & Não & 390 & 34,1 & 1 & & 1 & \\
\hline & Sim & 205 & 46,0 & $1,34(1,18-1,53)$ & & $1,11(0,96-1,29)$ & \\
\hline & HAS & & & & 0,000 & & 0,010 \\
\hline & Não & 220 & 31,0 & 1 & & 1 & \\
\hline & Sim & 375 & 42,6 & $1,37(1,20-1,57)$ & & $1,19(1,04-1,37)$ & \\
\hline & Diabetes & & & & 0,090 & & 0,171 \\
\hline & Não & 504 & 37,3 & 1 & & 1 & \\
\hline & Sim & 91 & 37,8 & $1,01(0,84-1,20)$ & & $0,87(0,72-1,05)$ & \\
\hline & Reumatismo/artrite/artrose & & & & 0,000 & & 0,000 \\
\hline & Não & 339 & 29,3 & 1 & & 1 & \\
\hline & Sim & 256 & 59,0 & $2,01(1,78-2,26)$ & & $1,67(1,46-1,91)$ & \\
\hline & Déficit Cognitivo & & & & 0,002 & & 0,000 \\
\hline & Sem déficit & 409 & 41,0 & 1 & & 1 & \\
\hline & Com déficit & 168 & 32,6 & $0,79(0,68-0,91)$ & & $0,72(0,61-0,85)$ & \\
\hline \multirow[t]{7}{*}{$4^{\circ}$ nível } & Capacidade funcional & & & & & & \\
\hline & ABVD & & & & 0,007 & & 0,153 \\
\hline & Sem dificuldade & 549 & 38,6 & 1 & & 1 & \\
\hline & Com dificuldade & 46 & 27,2 & $0,70(0,54-0,90)$ & & $0,79(0,58-1,08)$ & \\
\hline & AIVD & & & & 0,671 & & 0,493 \\
\hline & Sem dificuldade & 396 & 37,7 & 1 & & 1 & \\
\hline & Com dificuldade & 149 & 36,6 & $0,97(0,84-1,11)$ & & $1,05(0,90-1,22)$ & \\
\hline \multirow[t]{13}{*}{$5^{\circ}$ nível } & Utilização serviços de saúde & & & & & & \\
\hline & Hospit. Último ano & & & & 0,801 & & 0,145 \\
\hline & Não & 488 & 37,3 & 1 & & 1 & \\
\hline & Sim & 107 & 38,1 & $1,02(0,86-1,20)$ & & $0,88(0,74-1,04)$ & \\
\hline & Emergência 3 meses & & & & 0,089 & & 0,899 \\
\hline & Não & 508 & 36,7 & 1 & & 1 & \\
\hline & Sim & 87 & 42,6 & $1,16(0,97-1,38)$ & & $1,01(0,83-1,22)$ & \\
\hline & Domicílio 3 meses & & & & 0,958 & & 0,871 \\
\hline & Não & 574 & 37,1 & 1 & & 1 & \\
\hline & Sim & 21 & 46,7 & $1,00(0,78-1,29)$ & & $1,02(0,76-1,36)$ & \\
\hline & Consulta 3 meses & & & & 0,000 & & 0,008 \\
\hline & Não & 225 & 31,2 & 1 & & 1 & \\
\hline & Sim & 370 & 42,7 & $1,36(1,19-1,56)$ & & $1,20(1,05-1,38)$ & \\
\hline
\end{tabular}


por 3 ou mais horas, reduziu em 17,0\% (RP: 0,83; IC95\% 0,73-0,94) a probabilidade de DCV. Idosos com autoavaliação de saúde péssima/ruim apresentaram 1,95 vezes (RP: 1,95; IC95\% 1,602,38) mais ocorrência do desfecho pesquisado, em comparação àqueles com saúde boa e muito boa (Tabela 2).

Quanto as comorbidades, idosos com HAS (RP: 1,19; IC95\% 1,04-1,37) e reumatismo (RP: 1,67; IC95\% 1,46-1,91) apresentaram maior probabilidade de DCV quando comparados àqueles sem estas doenças. A presença de déficit cognitivo mostrou ser proteção para o desfecho (RP: 0,72; IC95\% 0,61-0,85). Com relação à utilização de serviços, consulta nos últimos três meses aumentou em 20,0\% a probabilidade de distúrbios nas costas (Tabela 2).

\section{Discussão}

O presente estudo analisou a prevalência de distúrbios na coluna e seus fatores associados em idosos da zona urbana do extremo Sul do Brasil. Os achados indicam uma elevada prevalência do desfecho, apresentando como fatores associados o sexo feminino, baixa escolaridade, auto avaliação ruim de saúde, consulta nos últimos três meses e presença de hipertensão, reumatismo e fraturas. Além disso, ser solteiro/separado ou viúvo, ter comportamento sedentário e apresentar déficit cognitivo mostrou proteção para a DCV.

A alta prevalência de $\operatorname{DCV}(37,4 \%)$ foi consistente com achados da literatura nacional e internacional, evidenciando a relevância do problema para a saúde pública entre os idosos, além de ser a principal causa de incapacidade em todo o mundo ${ }^{1,5,6}$.

À semelhança do presente estudo, pesquisas revelam a associação entre sexo feminino e distúrbios na coluna vertebral ${ }^{12-15}$. Estudo de revisão sistemática acerca da prevalência de dor lombar e dor nas costas oriundas de distúrbios nesta estrutura, sugere que a maior presença do desfecho entre as mulheres pode estar relacionada a diferentes fatores, como inicio precoce da puberdade que gera diferenças no crescimento entre os sexos, maior propensão fisiológica à osteopenia e osteoporose, alterações hormonais oriundas da menstruação e da menopausa, gravidez e diferenças comportamentais e sociais que resultam em maior probabilidade de apresentar $\mathrm{DCV}^{16}$.

Nossos achados também revelam que idosos solteiros ou viúvos apresentam menor probabilidade de distúrbios na coluna do que idosos casados ou que vivem com seus companheiros. Sabe-se que idosos que vivem sozinhos normalmente são mais saudáveis e independentes, o que os torna menos suscetíveis ao desfecho. Pesquisas nacionais sobre incapacidade funcional em idosos tem encontrado resultados semelhantes, e sugerem a relação entre viver sozinho e as questões psicológicas, físico-funcionais e financeiras atreladas a esta condição, que por sua vez acreditamos que também justifique a associação encontrada nesta pesquisa, independente da diferença entre os desfechos pesquisados ${ }^{17,18}$.

Com relação à escolaridade, ter nenhuma ou baixa escolaridade esteve associado a distúrbios na coluna, concordando com resultados de uma pesquisa de base populacional que revela que indivíduos com menor nível educacional e que residem em regiões menos favorecidas apresentam maiores probabilidade de sentir dor. Os autores sugerem que esses indivíduos estão expostos a maiores sobrecargas ergonômicas, seja em casa ou no trabalho, o que justificaria este resultado ${ }^{19}$.

Neste estudo, o comportamento sedentário mostrou ser fator de proteção para o desfecho pesquisado. A literatura apresenta consenso acerca dos malefícios do sedentarismo para a saúde cardiovascular e musculoesquelética ${ }^{20}$. Contudo, considerando que a distúbios na coluna vertebral em idosos pode estar vinculada ao desgaste natural sofrido pelas estruturas musculoesqueléticas. Acredita-se que a redução nas atividades diminui a dor nas costas ao proteger estruturas já fragilizadas pelo processo de envelhecimento do impacto e sobrecarga nestas. Cabe destacar que é difícil estabelecer com segurança relações causais entre distúrbios na coluna e comportamento sedentário nesta população, pois além da possibilidade de causalidade reversa oriunda do delineamento do estudo, devemos considerar o viés de sobrevivência, permanecendo vivos idosos mais saudáveis, nos quais o sedentarismo não tem o mesmo impacto do que na população mais jovem.

Também foi observado que quanto pior a percepção de saúde maior a prevalência de distúrbios na coluna. Pesquisas que estudam a relação entre depressão, auto percepção de saúde ruim e dor nas costas também encontram resultados semelhantes, uma vez que a auto percepção de saúde é um importante indicador das condições de saúde física, cognitiva, emocional e de mortalidade, associando-se fortemente com o estado objetivo de saúde dos indivíduos ${ }^{21,22}$.

De acordo com os resultados, idosos que sofreram fraturas apresentaram maior probabilidade de apresentar distúrbios na coluna verte- 
bral. Estas fraturas, por sua vez, levam o idoso a imobilidade, dependência, redução do trofismo muscular e da propriocepção articular, que consequentemente podem justificar o resultado encontrado nesta pesquisa ${ }^{23}$.

No que diz respeito às comorbidades, idosos com HAS e/ou reumatismo/artrite/artrose apresentaram maior probabilidade de desenvolver distúrbios na coluna. Este achado é corroborado por outros estudos pesquisados, os quais sugerem que as comorbidades podem levar a maior vulnerabilidade, que por sua vez poderão impactar nos resultados de saúde ${ }^{12,24}$. Em consonância, a artrite/artrose também justifica-se pela fonte nociceptiva que a disfunção articular facetária característica da morbidade causa na coluna vertebral ${ }^{12}$. Cabe ponderar que este resultado reforça a necessidade de modelos de cuidado aos idosos que considerem as multimorbidades para o desenvolvimento de estratégias de prevenção, promoção e tratamento.

De acordo com os dados, idosos com deficit cognitivo referiram menos distúrbios na coluna. Acreditamos que este resultado pode estar atrelado a metodologia de coleta dos dados, uma vez que as informações foram dadas por um informante chave, que pode não ter total conhecimento sobre a saúde musculoesquelética do ido- so. Por outro lado, idosos com deficit cognitivo podem apresentar estilo de vida menos exposto a situações de risco para o surgimento de DCV, o que justificaria o resultado encontrado ${ }^{25}$.

Quanto à associação entre o desfecho e consulta nos últimos 3 meses, a literatura refere que os problemas nas costas são um dos principais motivos de busca por atendimento médico ${ }^{26}$. Ao analisar este resultado é necessário valorizar a possibilidade de causalidade reversa, podendo os idosos que apresentam distúrbios na coluna serem justamente aqueles que buscam mais o serviço de saúde.

As evidências apresentadas neste estudo reforçam a importância das atividades de promoção e prevenção da saúde desenvolvidas no âmbito da Atenção Básica. A prevalência da distúrbios na coluna vertebral e alguns dos fatores associados verificados nesta pesquisa, como fratura e presença de comorbidades (HAS e reumatismo/artrite/ artrose), podem ser evitados através de intervenções focadas na manutenção da saúde das estruturas musculoesqueléticas, que por sua vez também reduzem os danos decorrentes dos distúrbios na coluna vertebral na população idosa, como comorbidades, perda de independência, aumento nas demandas para os serviços de saúde e para previdência social e redução na qualidade de vida.

\section{Colaboradores}

Os autores MO Saes, JDN Lopes e BP Nunes participaram da concepção, delineamento, análise, redação do artigo. SMS Duro, LA Facchini e E Thumé participaram da redação do artigo, revisão crítica e aprovação da versão a ser publicada. 


\section{Referências}

1. Green BN, Johnson CD, Haldeman S, Griffith E, Clay MB, Kane EJ, Castellote JM, Rajasekaran S, Smuck M, Hurwitz EL, Randhawa K, Yu H, Nordin M. A scoping review of biopsychosocial risk factors and co- morbidities for common spinal disorders. PLoS ONE 2018; 13(6):e0197987.

2. Meron A, Akuthota V. Spine Disorders in Older Adults. In: Cifu DX, Lew HL, Oh-Park M. Geriatric Rehabilitation. Berkeley: Elsevier; 2018. p.195-200.

3. Nunes BP, Thumé E, Facchini LA. Multimorbidity in older adults: magnitude and challenges for the Brazilian health system. BMC Public Health 2015; 15:1172.

4. Maher C, Underwood M, Buchbinder R. Non-specific low back pain. Lancet 2017; 389(10070):736-747.

5. Hartvigsen J, Hancock MJ, Kongsted A, Louw Q, Ferreira M, Genevay S, Hoy D, Karppinen J, Pransky G, Sieper J, Smeets RJ. What low back pain is and why we need to pay attention. Lancet 2018; 391(10137):23562367.

6. Leopoldino AAO, Bergamaschine JMD, Martinsb VT, Henschkec N, Pereira LSM, Dias RC, Oliveira VC. Prevalence of low back pain in older Brazilians: a systematic review with meta-analysis. Rev Bras Reumatol 2016; 56(3):258-269.

7. Romero DE, Santana D, Borges P, Marques A, Castanheira D, Rodrigues JM, Sabbadini L. Prevalência, fatores associados e limitações relacionados ao problema crônico de coluna entre adultos e idosos no Brasil. Cad Saúde Pública 2018; 34(2):e00012817.

8. Viniol A, Jegan N, Brugger M, Leonhardt C, Barth J, Baum E, Becker A, Strauch K. Even worse - risk factors and protective factors for transition from chronic localized low back pain to chronic widespread pain in general practice. Spine 2015; 40(15):E890-E899.

9. Makris UE, Fraenkel L, Han L, Leo-Summers L, Gill, TM. Epidemiology of Restricting Back Pain in Community-Living Older Persons. J Am Geriatr Soc 2011; 59(4):610-614.

10. Figueiredo VF, Pereira LS, Ferreira PH, Pereira AM, Amorim JS. Functional disability, depressive symptoms and low back pain in the elderly. Fisioter Mov 2013; 26(3):549-557.

11. Thumé E, Facchini LA, Tomasi E, Vieira LAS. Assistência domiciliar a idosos: fatores associados, características do acesso e do cuidado. Rev Saúde Pública 2010; 44(6):1-10.

12. Jiménez-Sáncheza $S$, Fernández-de-las-Penãs C, Carrasco-Garrido P, Hernández-Barrera V, Alonso-Blanco C, Palacios-Cenã D, Jiménez-García R. Prevalence of chronic head, neck and low back pain and associated factors in women residing in the Autonomous Region of Madrid (Spain). Gac Sanit 2012; 26(6):534540.

13. Ferreira GD, Silva MC, Rombaldi AJ, Wrege ED, Siqueira FV, Hallal PC. Prevalência de dor nas costas e fatores associados em adultos do Sul do Brasil: estudo de base populacional. Rev Bras Fisioter 2011; 15(1):3136.

14. Matos MG, Azevedo HE, Hoefel AL, Soares DJ. Dor lombar em usuários de um plano de saúde: prevalência e fatores associados. Cad Saúde Pública 2008; 24(9):2115-2122.
15. Bejia I, Younes M, Jamila HB, Khalfallah T, Salem KB, Touzi M, Akrout M, Bergaoui N. Prevalence end factors associated to low back pain among hospital staff. Joint Bone Spine 2005; 72(3):254-259.

16. Hoy D, Bain C, Williams G, March L, Brooks P, Blyth F, Woolf A, Buchbinder R. A Systematic Review of the Global Prevalence of Low Back Pain. Arthritis Rheumatism 2012; 64(6):2028-2037.

17. Sudré MRS, Reiners AAO, Nakagawa JTT, Azevedo RCS, Flori- ano LA, Morita LHM. Prevalence of dependency and associated risk factors in the elderly. Acta Paul Enferm 2012; 25:947-953.

18. Aguiar ARSA, Ribeiro-Samora GA, Pereira LSM, Godinho LB, Assis MG. Disability in older adults with acute low back pain: the study Back Complaints in the Elderly - (Brazil). Braz J Phys Ther 2017; 21(5):365371.

19. Silva MC, Fassa ACG, Valle NCJ. Dor lombar crônica em uma população adulta no Sul do Brasil: prevalência de fatores associados. Cad Saúde Pública 2004; 20(2):377-385.

20. Rezende LFM, Rey-López JP, Matsudo VKR, Luiz OC. Sedentary behavior and health outcomes among older adults: a systematic review BMC Public Health 2014; 14:333.

21. Borges LJ, Benedetti TRB, Xavier AJ, d'Orsi E. Fatores associados aos sintomas depressivos em idosos: estudo Epi Floripa. Rev Saúde Pública 2013; 47(4):701-710.

22. Fujii T, Oka H, Katsuhira J, Tonosu J, Kasahara S, Tanaka S, Matsudaira K. Association between somatic symptom burden and health-related quality of life in people with chronic low back pain. PLoS One 2018; 13(2):e0193208.

23. Vieira LS, Gomes AP, Bierhals IO, Farías-Antúnez S, Ribeiro CG, Miranda VIA, Lutz BH, Barbosa-Silva TG, Lima NP, Bertoldi AD, Tomasi E. Falls among older adults in the South of Brazil: prevalence and determinants. Rev Saude Publica 2018; 52:22.

24. Zullig LL, Bosworth HB, Jeffreys AS, Corsino L, Coffman CJ, Oddone EZ, Yancy Jr WS. The association of comorbid conditions with patient-reported outcomes in Veterans with hip and knee osteoarthritis. Clin Rheumatol 2015; 34(8):1435-1441.

25. Briggs AM, Cross MJ, Hoy DM, Sànchez-Riera L, Woolf AD, March L. Musculoskeletal Health Conditions Represent a Global Threat to Healthy Aging: A Report for the 2015 World Health Organization World Report on Ageing and Health. Gerontologist 2016; 56(S2):S243-S255.

26. Dionne CE, Von Kor M, Koepsell TD, Deyo RA, Barlow WE, Checkoway H. Formal education and back pain: a review. J Epidemiol Community Health 2001; 55(7):455-468.

Artigo apresentado em 31/10/2018

Aprovado em 29/04/2019

Versão final apresentada em 01/05/2019

Editores chefes: Romeu Gomes, Antônio Augusto Moura da Silva 
